\title{
COMMENT
}

Check for updates

\section{Health care for people who are incarcerated}

Lisa B. Puglisi ${ }^{1,2}$ and Emily A. Wang ${ }^{1,2 凶}$

Incarcerated individuals and those with a history of incarceration have high rates of medical conditions yet face several barriers to care. Removing these barriers through adequate funding, enhancing the role of community health systems, and addressing stigma and discrimination are imperative to improve the health and well-being of this population.

The interaction between incarceration and health is complex, especially in countries without universal health care

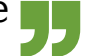

'SEICHE Center for Health and Justice, Yale School of Medicine, New Haven, CT, USA.

${ }^{2}$ Department of Internal Medicine, Yale School of Medicine, New Haven, CT, USA.

凶e-mail:

emily.wang@yale.edu

https://doi.org/10.1038/

s41572-021-00288-9
Worldwide, an estimated 11 million people are incarcerated in correctional facilities. In the USA, they are held in two different systems: jails, which detain people before adjudication of a crime and those with short sentences; and prisons, which detain those with sentences $>1$ year ${ }^{1}$. Although its incarceration rate is at the lowest level since 2000 , the USA has the highest prison population globally, with 2.1 million individuals incarcerated at any given time (see World Prison Brief - Prison Population Total). Globally, correctional systems are disproportionately filled with individuals of low socioeconomic status and religious, ethnic and racialized minorities (targets of exclusionary social and economic policy) ${ }^{2}$.

Incarcerated people have high rates of chronic health conditions, including communicable diseases (such as sexually transmitted infections and tuberculosis) and non-communicable diseases (such as asthma, hypertension, mental health and substance use disorders) ${ }^{3}$. The interaction between incarceration and health is complex, especially in countries without universal health care. Incarceration in the USA may improve the status of chronic conditions such as HIV and can lead to new diagnoses of preexisting health issues such as hepatitis $\mathrm{C}$, as some incarcerated individuals gain access to health care ${ }^{4}$. In addition, being away from communities with high levels of gun violence is associated with decreased mortality. By contrast, conditions of confinement - the built environment, crowding, social isolation, inactivity, and poor air and water quality - can worsen health. Indeed, a history of incarceration is associated with increased rates of chronic health conditions, such as hypertension, diabetes mellitus and asthma, during incarceration and after release. This Comment primarily discusses incarceration in the USA as the most extreme example of mass incarceration and its effect on health (FIC. 1).

Native Americans and incarcerated people are the only Americans who have a constitutional right to health care; however, a constitutional guarantee to care does not ensure quality or equivalent access. Most people receive a basic health screening upon jail entry and correctional facilities vary from offering urgent care to hospital equivalent care. In general, preventive care and chronic disease management are limited; for instance, most prisons and jails do not provide opt-out hepatitis $C$ screening or have medications for opioid use disorder, conditions that are highly prevalent in this population. Indeed, no mandatory standards and oversights exist for correctional health care. The largest systematic changes in prison health care take place due to litigation.

Some incarcerated individuals have unique health-care needs, including people who are pregnant, transgender and elderly. Over the past 40 years, the number of incarcerated women increased sevenfold, and an estimated 3-5\% are pregnant upon detainment. ${ }^{5}$ Transgender individuals are over-represented in carceral settings, where they are disproportionately exposed to violence, abuse and solitary confinement and have limited access to gender-affirming care ${ }^{6}$. The age of people in prison and jails has also considerably increased and geriatric care needs are a growing focus; for instance, in 2013, people aged $\geq 55$ accounted for $10 \%$ of state prison populations ${ }^{7}$.

Health-care provision in correctional systems is challenging. Even providing routine and preventive care can be difficult, especially in jails, which have high throughput and short lengths of stay, and in rural facilities, which have limited access to specialty care. Correctional health care is further complicated by a complex interplay of factors, including the logistics of movement within the facility, transportation to and from external health facilities, and health-care provider shortages, that constrain health-care delivery, as well as the institutional stigma of addiction and procedures such as shackling during childbirth or chemotherapy that fracture patient trust. Some emerging evidence shows that both screening-detectable and non-screening-detectable cancers are diagnosed at more advanced stages in incarcerated individuals than in the general population, and that distance to treatment centers and cost of cancer care provide substantial challenges to providing guideline-concurrent care ${ }^{8}$. Also, correctional systems vary widely in approaches to screening for and treatment of hepatitis $\mathrm{C}$, for example, owing to the cost of treatment with direct acting antivirals. 
Pre-incarceration

\begin{tabular}{|c|c|}
\hline Structural & $\begin{array}{l}\text { - Socioeconomic position } \\
\text { - Racism } \\
\text { - Exposure to violence } \\
\text { - Educational attainment } \\
\text { - Exposure to police stops }\end{array}$ \\
\hline Health-care & $\begin{array}{l}\text { - Limited/no access to primary } \\
\text { care and insurance } \\
\text { - High use of emergency } \\
\text { department }\end{array}$ \\
\hline Behavioral & $\begin{array}{l}\text { - Smoking and substance use } \\
\text { - Poor diet and low physical } \\
\text { activity } \\
\text { - High-risk sexual behaviour }\end{array}$ \\
\hline Psychosocial & $\begin{array}{l}\text { - Adverse childhood experiences } \\
\text { - Family/social ties } \\
\text { - Perceived discrimination } \\
\text { - Housing or food insecurity }\end{array}$ \\
\hline
\end{tabular}

\section{Incarceration}

- Exposure to incarceration policies, institutional violence and trauma

- Guaranteed shelter and food

- Congregate living quarters and low-quality food

- Overcrowding

- Correctional oversight of healthcare

- Guaranteed healthcare

- Monitored medication adherence

- Newly diagnosed chronic disease

- Limited access to medications for addiction

- Correctional system involvement in health care

- Co-payments exceeding many days worth of work

- Limited access to smoking, alcohol and other drugs

- Little control over diet and variable physical activity

- Limited access to condoms

- Acute and chronic stress

- Social isolation

- Lack of autonomy or low self-efficacy

Re-incarceration $\uparrow$
Post-incarceration

- Lower socioeconomic position

- Racism

- Exposure to violence

- Collateral consequences

- Parole and probation supervision

- - - -

Limited/no access to primary care and insurance

$\rightarrow$ - Poor transitions in care

- Medical system discrimination

- High use of emergency department

Return to smoking and substance use

- More control over diet and physical activity

- High-risk sexual behaviour

- Acute and chronic stress

- Improved self-efficacy

- Altered family or social ties

- Perceived discrimination

- Housing or food insecurity

Fig. 1 | Factors associated with health in individuals who are incarcerated. Structural, behavioural and social factors, in addition to health-care factors, are associated with health in incarcerated individuals.

Another challenge for the delivery of health care for incarcerated individuals is that many correctional systems require high co-payments for health care. Given the ageing of the incarcerated population and high rates of chronic illness, health-care costs can consume up to $25 \%$ of state correctional budgets and many systems resort to cost-cutting strategies. As of 2015, 20 states subcontracted health-care delivery, often to for-profit companies?. In addition, most of the health system of correctional systems is entirely separate from that of the general community. Incarcerated people are, therefore, often released from correctional facilities without health insurance, medical records, sufficient supplies of medications, or a primary-care appointment, whilst also contending with severe structural barriers to obtaining housing, food, employment and primary care. Moreover, the $40 \%$ of individuals newly diagnosed with a chronic health condition while incarcerated must learn how to manage their conditions in the community setting, including use of pharmacies and managing medications. Unsurprisingly, they have high rates of hospitalizations and death immediately following release from conditions that may be preventable with continuity of care, including overdose, cardiovascular disease and cancer ${ }^{10}$.

With the large cluster outbreaks of COVID-19 in prisons and jails, there has been more of an urgent focus on the role of decarceration, namely limiting new incarcerations and expediting releases, as a strategy to mitigate COVID-19 transmission within facilities. Health-care providers and policy makers have many opportunities to improve the health care of people who experience incarceration, both during their detainment and after release. The biggest opportunities lie with expanding public health insurance to cover care for incarcerated people and expanding the role of the community health system to provide care that can interrupt cycles of incarceration by addressing upstream drivers and downstream health effects.
At the patient level, correctional systems must discharge people with sufficient medications and training to use them correctly, as well as health records and a primary-care appointment. In addition, community health systems can eliminate barriers to care, such as removing the requirement that patients show photo identification at their first appointment (as many people leaving correctional systems do not have identification) and providing cell phones and training on how to use them for telemedicine.

System-wide restructuring could include in-reach into correctional facilities to engage with incarcerated people before their release, low-barrier treatment of addictions, and primary care staffed with community health workers with histories of incarceration who build trust and address the social determinants of health after release. Efforts to dismantle discrimination in health systems and create health equity must attend to the unique needs of this patient population.

1. Walmsley R. World prison population list (Home Office, London, 2003).

2. Jacobson J. et al. Prison: Evidence of its Use and Over Use from Around the World (University of London, 2017)

3. Wilper, A. P. et al. The health and health care of US prisoners: results of a nationwide survey. Am. J. Public Health. 99, 666-672 (2009).

4. Meyer, J. P. et al. Optimization of human immunodeficiency virus treatment during incarceration: viral suppression at the prison gate. JAMA internal medicine. 174, 721-729 (2014).

5. Sufrin, C. et al. Pregnancy outcomes in US prisons, 2016-2017. American journal of public health. 109, 799-805 (2019).

6. Brömdal A. et al. Whole-incarceration-setting approaches to supporting and upholding the rights and health of incarcerated transgender people. Int. J. Transgend. 20, 341-350 (2019).

7. Carson E. A. et al. Aging of the state prison population, 1993-2013. Report No. 248766. Bureau of Justice Statistics, US Department of Justice (2016).

8. Sunthankar, K. I. et al. Cancer stage at presentation for incarcerated patients at a single urban tertiary care center. PLOS ONE 15 e0237439 (2020).

9. Mattick R. P. et al. Methadone maintenance therapy versus no opioid replacement therapy for opioid dependence. Cochrane Database Syst. Rev. 2009, CD002209 (2009).

10. Binswanger, I. A. et al. Release from prison-a high risk of death for former inmates. N. Engl. J. Med. 356, 157-165 (2007).

\section{Competing interests}

The authors declare no competing interests. 